\title{
Quantification du transport littoral par traçages fluorescents - Application au site de la Péninsule d'Ancão, Sud Portugal.
}

\author{
Yann Balouin ${ }^{1}$, Hélène Howa ${ }^{2}$ et Amaury De Resseguier ${ }^{3}$. \\ 1 ATER, UMR CNRS-5805-EPOC, Université Bordeaux 1, Av. des Facultés, \\ 33405 Talence Cedex, v.balouin@epoc.u-bordeaux.fr. Tel:05 56848438 \\ 2 Maître de conférences, Université d'Angers, UPRES EA 2644, \\ Boulevard Lavoisier, 49045 Angers Cedex. helene.howa@univ-angers.fr \\ ${ }^{3}$ Ingénieur d'études, UMR CNRS 5805, DGO, Université Bordeaux 1, \\ Av. des Facultés, 33405 Talence Cedex.
}

Résumé

L'objectif de cette présentation est l'étude du transport sédimentaire littoral, et en particulier, la quantification du sédiment transporté par la dérive littorale. Le site d'étude est la Plage de Faro sur la péninsule d'Ancão (Portugal), localisée en amont de l'embouchure de la Barra Nova. L'objectif de ce travail était la quantification de la contribution de la côte amont au bilan sédimentaire de l'embouchure. Les trois étapes de cette étude sont: i) les traçages fluorescents, associés à des mesures hydrodynamique, ii) la validation de formules de transport, iii) la contribution de la morphodynamique à la compréhension du système.

\section{Abstract}

The aim of this work is to progress in the knowledge of sediment circulation in the coastal zone, focusing on the quantification of sediment movement due to longshore drift. The studied field site is the Praia de Faro beach on the Peninsula do Ancão in Algarve (Portugal), located on the updrift coast of the Barra Nova tidal inlet. The main objective of the study was to quantify the sediment transport rate that will enter in the inlet system. The main steps of the study are i) tracer experiments, associated with hydrodynamic measurements, ii) longshore transport fomula validation, iii) morphodynamics contribution.

\section{Introduction}

Afin de comprendre les processus affectant un linéaire côtier, il est nécessaire de quantifier les apports sédimentaires, leurs origines et les lieux de dépôts de ces stocks. De manière générale, les bilans sédimentaires concernent les taux et particularités du transport sédimentaire à long terme. Dans le cas des côtes à embouchures, le bilan sédimentaire est indispensable à la compréhension de 
l'influence de ces systèmes sur les processus côtiers, et du comportement et de la stabilité de ces côtes largement observées dans le monde.

Dans le cadre du programme MAST III - INDIA (Williams et al., 1998), l'embouchure de la Barra Nova en Algarve, au sud du Portugal (fig. 1) a fait l'objet d'une campagne de mesures intensive. Les embouchures de la Ria Formosa migrent habituellement vers l'est, jusqu'au comblement progressif du chenal et la fermeture définitive (Pilkey et al., 1989). La raison principale de cette migration est une forte dérive littorale d'ouest en est alimentée par l'érosion des falaises situées à l'ouest (Dias and Neal, 1992).

L'objectif de cette étude était de quantifier le transport sédimentaire longshore le long de la péninsule d'Ancao afin de connaître les entrées dans le bilan sédimentaire de l'embouchure de la Barra Nova (fig. 1). Dans ce but, deux approches ont été suivies: i) le déploiement d'appareil in-situ qui est souvent lourd, coûteux et long, mais absolument nécessaire; ii) la validation de modèles qui permet d'intégrer et d'extrapoler les connaissances de terrain.

\section{Site d'étude}

La campagne de mesure a eu lieu de janvier à mars 1999 le long de la péninsule d'Ancao sur le site de la plage de Faro, sur la côte amont de la Barra Nova (fig. 1). Cette plage étroite est très réflective. Le sédiment est constitué de sables moyens $\left(\mathrm{D}_{50} \sim 0,5 \mathrm{~mm}\right)$. Sur cette côte orientée NW-SE, le marnage est mésotidal (entre 2 et 3,8 m) (Granja et al., 1984). La houle, principalement d'ouest, a une hauteur variant entre 1 et $4 \mathrm{~m}$ (78\% des vagues mesurent de $0-1 \mathrm{~m})$ (Pires, 1998), et génère un important transport "longshore" le long de la péninsule.

Les conditions hydrodynamiques ont été enregistrées à l'aide de plusieurs courantomètres, marégraphes, capteurs de pression. L'évolution morphologique a été suivie à l'aide de levés topographiques réguliers. Le transport sédimentaire résiduel (sur un cycle tidal) a été quantifié par traçage fluorescent. Le traceur est constitué de sable local marqué par de la peinture fluorescente rouge. Ce traceur est immergé à marée basse en bas de la zone intertidale et sa dispersion est détectée lors des marées basses suivantes à l'aide du détecteur-compteur automatique (Howa et De Resseguier, 1994). Deux traçages ont été réalisés (12 et 28/02/1999) le long de la péninsule d'Ancão (fig. 1).

\section{Méthodes}

\section{1. Taux de transport basé sur les traçages fluorescents}

Le suivi de sédiments marqués pour déterminer le transport littoral in-situ est utilisé avec succès depuis les années cinquante (Zenkovitch, 1958). De nombreuses études ont utilisé cette technique, comme par exemple Duane and James (1980), Kraus et al. (1982), Ciavola, 1997, ...

Cette approche requiert deux conditions: i) le matériel utilisé doit avoir les mêmes propriétés que le sédiment naturel, ii) la détection du mouvement du traceur doit 


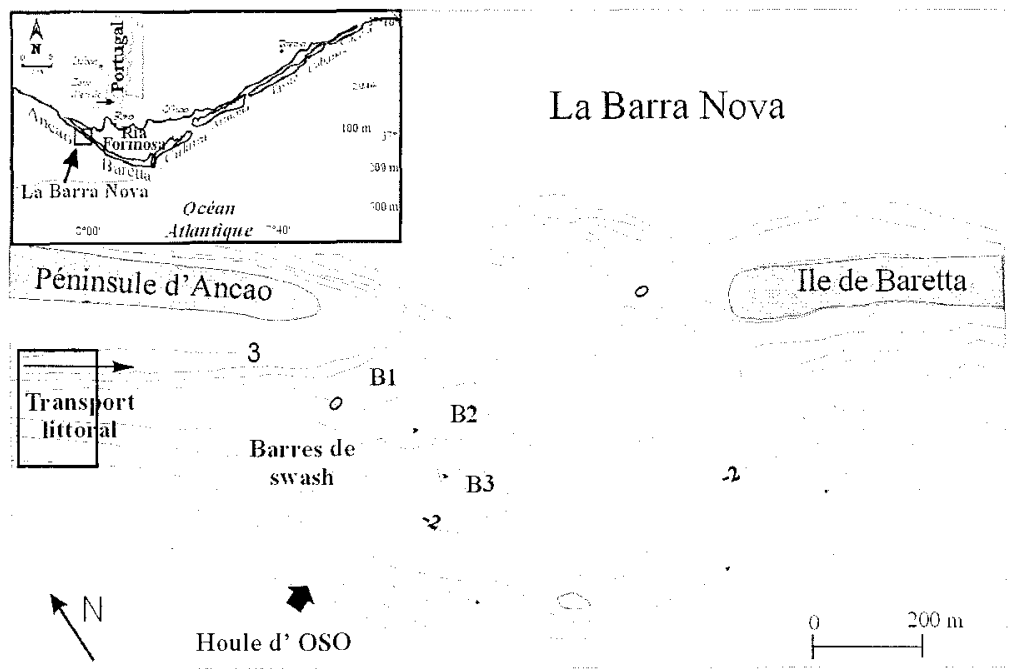

Figure 1: Contexte de l'étude: l'embouchure de la Barra Nova et sa localisation sur le système d'îles-barrière de la Ria Formosa. Context of the study: the Barra Nova inlet and its location on the Ria Formosa barrier islands system.

être précise. La $1^{\text {ère }}$ condition est remplie lorsque le traçage n'altère pas les propriétés mécaniques et physiques du sable, et la $2^{\text {nde }}$ est contrôlée en évaluant le taux de recouvrement. L'interprétation du traçage est basée sur le modèle physique de "rivière de sédiment" qui suppose que le sable est transporté en une couche uniforme d'épaisseur $Z_{0}$ à la vitesse $U$. Le taux de transport $(I, N / \mathrm{m} / \mathrm{s})$ est donné par:

$$
I=\left(\rho_{s}-\rho\right) \cdot g \cdot Q=\left(\rho_{s}-\rho\right) \cdot g \cdot N_{0} \cdot U \cdot Z_{0}
$$

où $\mathrm{Q}$ est le taux de transport volumétrique, $\rho_{\mathrm{s}}$ et $\rho$ les masses volumiques du sable et de l'eau. $\mathrm{N}_{0}$ est la concentration volumique du sable. La vitesse, $\mathrm{U}$, est déterminée par le déplacement du centre de gravité du nuage du traceur fluorescent, divisé par le temps d'immersion entre deux détections.

Le taux de recouvrement, contrôle de qualité du traçage, $R(\%)$, est donné par :

$$
R=\frac{100 \cdot M_{D}}{M_{T}-M_{R}}
$$

où $M_{T}$ est la masse totale du traceur, $M_{R}$ est la masse de traceur restant au point d'immersion et $M_{D}$ est la masse détectée sur la zone.

Un point critique de la méthode est l'estimation de l'épaisseur de la couche mobile (White, 1998). Celle-ci est obtenue par des carottages courts, en déterminant la concentration de traceur dans la carotte, $n$, et en appliquant le rapport de White and Inman 1989 :

$$
Z_{0}=\frac{\sum n(z) \Delta z(z)}{N_{\max }}
$$

où $\Delta \mathrm{z}$ est l'épaisseur d'une couche, $\mathrm{N}_{\max }$ la concentration maximale de traceur dans la carotte. De plus, un second marqueur est utilisé comme témoin en plusieurs points de la zone pour avoir une estimation supplémentaire de la couche mobile.

\section{3 - 2 - Calcul théorique du transport sédimentaire "longshore"}

La plupart des formules de prédiction du transport littoral sont basées sur des données de traçages (Schoonees and Theron, 1993). Quatre formules de transport 
(CERC, 1984; LCHF, 1979; Bailard, 1984; Kamphuis, 1991) ont été confrontées aux données de traçage afin de valider un outil prédictif valable dans cet environnement.

\section{Résultats}

\section{1 - Conditions hydrodynamiques}

Pendant la campagne, les conditions hydrodynamiques sont restées relativement calmes, avec des vagues d'W $\left(241-284^{\circ} \mathrm{N}\right)$, de hauteur significative $\left(\mathrm{H}_{\mathrm{s}}\right)$ et de période $\left(\mathrm{T}_{\mathrm{s}}\right)$ allant de 0,3 à $1 \mathrm{~m}$ et 4 à $6 \mathrm{~s}$. Ces conditions, constantes sur la durée de l'expérience, sont représentatives des conditions estivales qui prédominent dans la région (plus de $80 \%$ du temps). Les courants littoraux ont été mesurés en continu pendant un mois. L'hydrodynamique observée lors des traçages est représentée sur la figure 2 qui illustre les faibles variations de la houle lors des cycles tidaux étudiés. L'impact de la marée sur la dérive littorale est mineur.

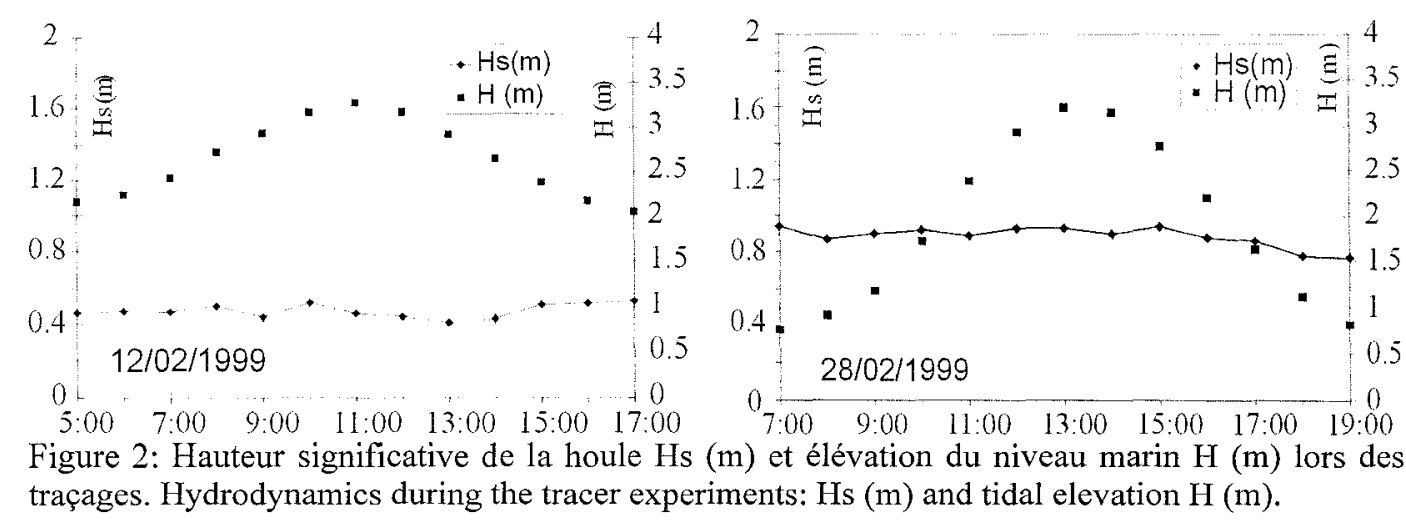

\section{2 - Analyse du traçage}

Deux expériences ont été réalisées, les 12 et 28/02/99 (tableau 1). La première a eu lieu dans une zone où la pente de la plage est forte $(0,11)$, et sa largeur d'environ $30 \mathrm{~m}$ (fig. 4). L'épaisseur de remaniement était de $13 \mathrm{~cm}$ et presque tout le traceur a bougé. La quantité de traceur détecté permet de calculer un taux de recouvrement de $64 \%$, ce qui autorise la quantification du transport. Le mouvement du traceur donne un taux de transport résiduel de $87,16.10^{-6} \mathrm{~m}^{3} / \mathrm{s} / \mathrm{m}$, ou $2,61.10^{-3} \mathrm{~m}^{3} / \mathrm{s}$ en intégrant sur la largeur de la zone intertidale.

Le second traçage a été réalisé plus près de l'embouchure, où la pente de la plage est plus faible $(0,05)$ et la zone intertidale plus large. Les conditions de vagues étaient légèrement plus dynamiques $\left(\mathrm{Hs}_{\mathrm{s}}=0,8-1 \mathrm{~m}\right)$. L'épaisseur de remaniement était de l'ordre de $8 \mathrm{~cm}$, et le taux de recouvrement de 68,5\%. Le taux de transport résiduel obtenu est $68,44.10^{-6} \mathrm{~m}^{3} / \mathrm{s} / \mathrm{m}$, ou $4,24.10^{-3} \mathrm{~m}^{3} / \mathrm{s}$ en intégrant sur la plage. - Calcul théorique du taux de transport:

Les formules ont été utilisées pour calculer les taux de transport littoral, en utilisant les enregistrements effectués lors des expériences. Ces conditions de 


\begin{tabular}{|c|c|c|c|c|c|c|c|c|c|c|c|c|}
\hline \multirow[t]{2}{*}{ Traçage } & \multicolumn{2}{|c|}{$\begin{array}{l}\text { coordonnées du } \\
\text { centre de masse }\end{array}$} & \multirow{2}{*}{$\begin{array}{c}\text { distance } \\
d \\
(\mathrm{~m})\end{array}$} & \multirow{2}{*}{$\begin{array}{c}\text { épaisseur } \\
\mathrm{Zt} \mathrm{(m)}\end{array}$} & \multirow{2}{*}{$\begin{array}{c}\text { couche } \\
\text { mobile } \\
\mathrm{ZO}(\mathrm{m})\end{array}$} & \multicolumn{3}{|c|}{$\begin{array}{c}\text { vitesse } \\
\mathrm{m} / \mathrm{s} * 10-6\end{array}$} & \multicolumn{3}{|c|}{$\begin{array}{c}Q \\
(m 3 / s / m * 10-6)\end{array}$} & \\
\hline & $Y(m)$ & $\mathrm{X}(\mathrm{m})$ & & & & Vy & $V_{x}$ & IVI & Qy & $Q x$ & $|Q|$ & \\
\hline & & & & 0 & & & & & & 58.4 & 87.16 & 64.2 \\
\hline 281 & -23.8 & 23.002 & 33.106 & 0.01 & 0.08 & -1025 & 990.6 & 1425.7 & 49.22 & 47.55 & 68.44 & 8.5 \\
\hline
\end{tabular}

Tableau 1: Résultats des traçages. Tracer experiments results

houle étaient pour le $12 / 02: \operatorname{dir}=270^{\circ}, \mathrm{T}=4 \mathrm{~s}, \mathrm{Hs}=0,5 \mathrm{~m}$, et pour le $28 / 02: \operatorname{dir}=245^{\circ}$, $\mathrm{T}=5 \mathrm{~s}, \mathrm{Hs}_{\mathrm{s}}=0,8 \mathrm{~m}$. Les résultats sont présentés sur la fig 3 .

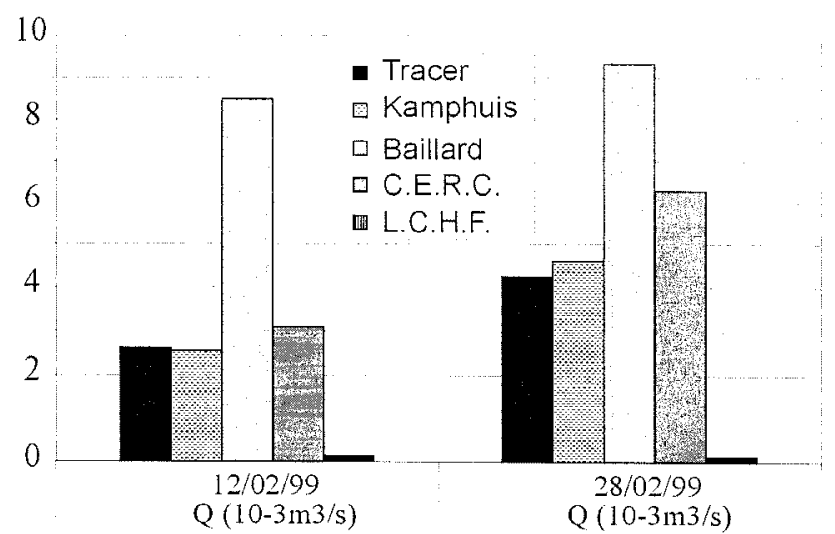

Figure 3: comparaison des taux de transport intégrés mesurés et prédits. Comparison of predicted and measured longshore transport.

Le taux de transport est largement sous-estimé avec la formule du L.C.H.F. Il est surestimé d'un facteur 2-3 par la formule de Bailard et variable par celle du CERC $(\mathrm{K}=0,77)$. Pour les deux traçages, la formule de Kamphuis donne des valeurs comparables aux données de terrain. Cette formule a donc été choisie pour estimer le transport sur différentes périodes de temps. On obtient, pour le mois de février 1999 un taux de transport de $10500 \mathrm{~m}^{3}$.

\section{3 - Interprétation morphologique}

Selon la classification de Masselink and Short (1993), les plages de la péninsule d'Ancão (fig. 4) sont du type "Low tide Terrace and rip", avec un comportement réflectif. La majorité du transport sédimentaire sur ce type de morphologie s'effectue sur la pente de la berme, une bande étroite où la majorité de l'énergie de la houle agit.

La partie amont du delta de jusant de la Barra Nova est constituée de barres sableuses, qui forment une plate-forme de swash qui s'étend sur 300-400 m vers le large (fig. 1) (Balouin et al., 2001). La première barre B1 est accolée à la berme. Les barres B2 et B3 sont des barres de swash qui constituent le corps de la plateforme. B1 est directement connectée à la pente de la berme et exclusivement alimentée par la dérive littorale en raison de la présence du delta qui réduit considérablement l'énergie incidente de la houle. Le volume de cette barre a augmenté très rapidement $\left(11000 \pm 500 \mathrm{~m}^{3}\right.$ en 1 mois $)$. Elle a migré de plus de $60 \mathrm{~m}$ en 1 mois (fig. 5). Les propriétés du sédiment sur cette barre sont les mêmes que sur les plages de la péninsule $\left(D_{50} \sim 0.5 \mathrm{~mm}\right.$, relativement homogène). 


\section{VII ${ }^{\text {emes }}$ Journées Nationales Génie Civil-Génie Côtier, Anglet, France, 15-17 Mai 2002}

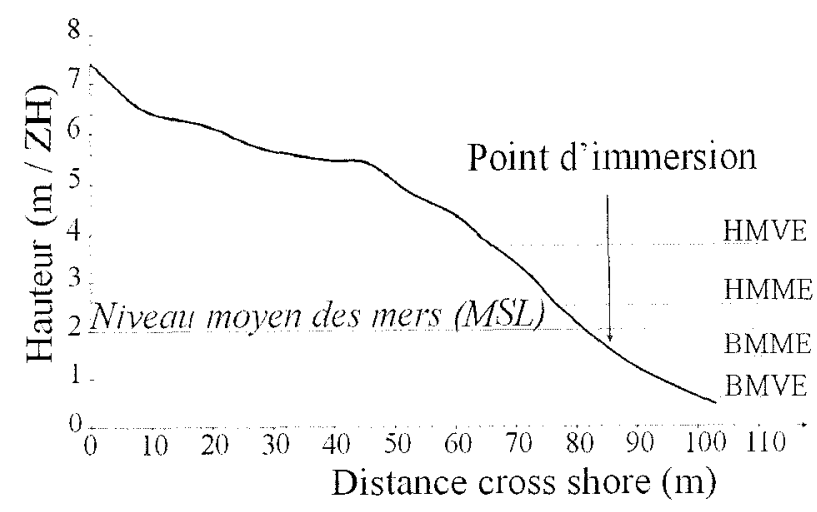

Figure 4: Profil de la plage sur la péninsule d'Ancão et localisation du point d'immersion $\mathrm{du}$ traceur fluorescent.

Cross shore transect of Ancão Peninsula and location of the immersion point.

\section{Discussion}

Du sable fluorescent a été immergé pour quantifier le taux de transport sédimentaire à court terme pour des conditions de vagues données. Les taux de transport "longshore" obtenus sont de $2,61.10^{-3}$ et $4,24.10^{-3} \mathrm{~m}^{3} / \mathrm{s}$. Pour des conditions similaires à Culatra, Ciavola et al. (1997) trouvaient un transport de $2,3.10^{-3}, 1,38.10^{-2}$ et $2,3.10^{-3} \mathrm{~m}^{3} / \mathrm{s}$ lors de 3 marées successives. Les taux relativement élevés obtenus lors de nos traçages semblent donc être habituels dans cette région où les épaisseurs de remaniement sont importantes. Ces résultats ont permis de tester les formules de transport les plus utilisées. La formule de Kamphuis semble être la plus adaptée à l'environnement de la plage de Faro, et son application pour les conditions hydrodynamiques du mois de février 1999 donne un taux de transport "longshore" de l'ordre de $10500 \mathrm{~m}^{3} /$ mois vers l'embouchure de la Barra Nova. Les variations de volume de la barre B1 sont de $11000 \mathrm{~m}^{3} /$ mois (Balouin et al., 2001), ce qui correspond à la valeur de la dérive littorale, et valide les taux de transport quantifiés.

Cette estimation du transport lors de conditions de "beau temps" s'avère très intéressante pour plusieurs raisons:

- d'une part, ces conditions, typique du climat estival d'WSW, représentent $80 \%$ des conditions annuelles,

- d'autre part, l'objectif de cette étude était de connaître la contribution de la côte amont au bilan sédimentaire de la Barra Nova. Or, on sait que la construction du delta de jusant s'effectue majoritairement lors de ces conditions calmes, et qu'il peut être complètement détruit lors des tempêtes.

- l'embouchure, à long terme migre vers l'est. Cette migration s'effectue majoritairement lors de tempêtes qui entraînent la destruction de l'île de Barreta sans toutefois générer un "saut" de l'embouchure (Balouin and Howa, 2001). Ces tempêtes sont suivies d'un état de transition pendant lequel la morphologie de tempête s'estompe progressivement, entraînant l'élargissement du chenal. Le système s'ajuste alors à la nouvelle section par une progradation de la péninsule d'Ancão, totalement liée à la dérive de beau temps.

Pour vérifier cette hypothèse d'ajustement après les tempêtes, le modèle analytique de progradation d'une flèche contrainte par un chenal (Kraus, 1999) a été utilisé. Le transport littoral annuel a été calculé à l'aide des données mesurées lors des années 1998-99, et des données enregistrées depuis 25 ans dans la région 


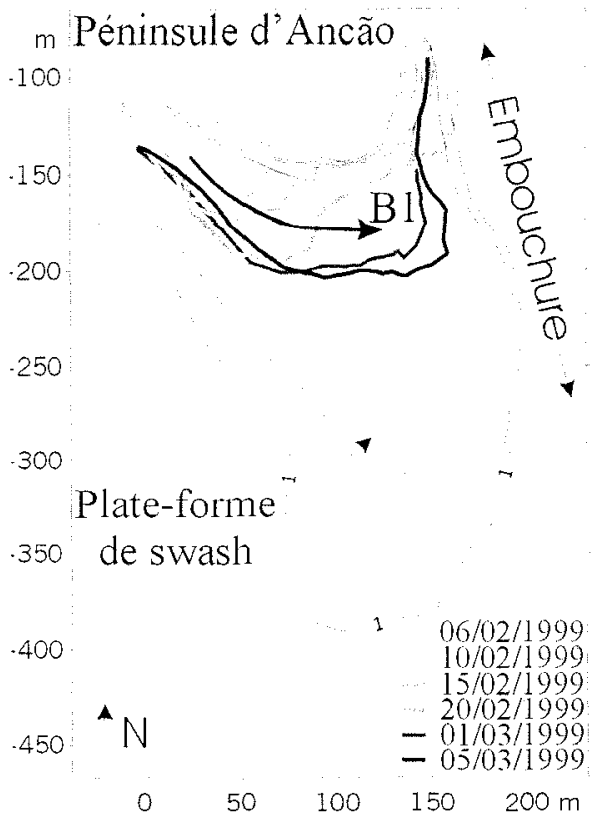

Figure 5: Evolution morphologique de la barre B1 sur la plate-forme de swash amont de l'embouchure. Morphological evolution of the Bar Bl located on the updrift swash platform of the ebb-tidal delta.

(Pires, 1998). L'application du taux de transport annuel (130000 $\left.\mathrm{m}^{3} / \mathrm{an}\right)$ dans le modèle de Kraus donne une progradation annuelle de la flèche d'Ancão de l'ordre de 60-70 m, soit une migration de 60-70m/an de la Barra Nova. Différents auteurs ont estimé le taux de migration de cette embouchure. Vila et al. (1999) ont montré, d'après des photographies aériennes que la valeur moyenne de 1976 à 1989 était de 1'ordre de 70-80 m/an. Morris et al. (2001) ont déterminé, par un suivi vidéo (14 mois), que la migration actuelle de la Barra Nova est de $70 \mathrm{~m} / \mathrm{an}$.

Ce calcul du taux de migration utilisant le taux de transport littoral quantifié par les traçages valide une fois de plus la méthodologie suivie et son intérêt dans la prédiction de l'évolution de ce type d'environnement.

\section{Conclusion}

L'objectif de cette étude était la quantification du transport littoral le long de la péninsule d'Ancão. Ce transit sédimentaire O-E alimente l'embouchure de la Barra Nova et représente un élément clé du bilan sédimentaire de ce système.

Des traçages fluorescents ont été réalisés, et les résultats validés par des mesures complémentaires d'hydrodynamique et de morphologie ont permis de valider un outil prédictif afin d'extrapoler nos mesures. Le formule de Kamphuis, connue pour être bien adaptée aux environnements à énergie modérée, reproduit de manière très satisfaisante les résultats de traçages, alors que les autres formules, largement utilisées, ont tendance à surestimer ce taux de transport, démontrant les limites de l'utilisation de ces outils sans calibration appropriée.

La méthodologie utilisée présente de nombreux avantages : reproductibilité, faible coût, impact environnemental faible et la possibilité d'application en « routine ». Cette étude montre que si les traçages sont connus pour donner de bons résultats à court-terme et pour des conditions hydrodynamiques données, une bonne 
connaissance du contexte environnemental permet une extrapolation à long terme rapide, facile et suffisamment précise pour des objectifs d'ingénierie côtière.

\section{7. $\underline{\text { Remerciements }}$}

Cette étude a été réalisée dans le cadre du programme MAST III - INDIA et financée par la communauté européenne (contrat MAST n MAS3-CT-0106).

\section{Références}

Bailard, J. A. 1984. "A Simplified Model for Longshore Sediment Transport," Proc. 19th International Coastal Engineering Conf., ASCE, New York, pp 1454- 1470.

Balouin, Y., Howa, H. and Michel, D., 2001. Swash platform morphology in the ebb-tidal delta of the Barra Nova Inlet, South Portugal. J. of Coastal Res 18 (3): 883-904.

Balouin, Y. and Howa, H., 2001. Sediment transport pattern at the Barra Nova inlet south Portugal: a conceptual model. Geo-marine Letters, in press.

Ciavola, P., Taborda, R., Ferreira, O., Dias, A., 1997. Field measurements of longshore sand transport and control processes on a steep meso-tidal beach. J. Coastal Res., 13(4): 1119-1129.

CERC, 1984.Shore Protection Manual 4th ed., 2 Vol, U. S. Army Engineer Waterways Experiment Station, U. S. Government Printing Office, Washington, DC.

Dias, J.M.A. and Neal, W.J., 1992. Sea cliff retreat in southern Portugal: Profiles, Processes and problems. Journal of Coastal Research, V. 8 -3, pp. 641-654.

Duane, D.B. and James, W.J., 1980. Littoral transport in the surf zone elucidated by an eulerian sediment tracer experiment. Journal of Sedimentary Petrology, v. 50, pp. 929-942.

Granja, H., Froidefond, J.M., Pera, T., 1984. Processus d'évolution morpho-sédimentaire de la Ria Formosa (Portugal). Bull. Inst. Géol. Bassin d'Aquitaine, Bordeaux, France, 254 pp.

Howa, H. and De Resseguier, A., 1994. Application of a fluorescent grain detector/counter for sand transport evaluation in the littoral zone. Proc. of Ocean OSATES 94, v. 3, pp.254-257.

Kamphuis, J. W. 1991. "Alongshore Sediment Transport Rate," Journal of Waterway, Port, Coastal, and Ocean Engineering, 117(6), pp 624-640.

Kraus, N.C., 1999. Analytical model of spit evolution at inlets. Proc. of Coastal Sediments 99, ASCE, 1999, New York, Long Island, pp. 1739-1754.

Kraus, N. C., Isobe, M., Igarashi, H., Sasaki, T. O., Horikawa, K. 1982. Field experiments on longshore sand transport in the surf zone, Proc. 18 ${ }^{\text {th }}$. Conf. Coastal Engineering, ASCE, 969-988.

Masselink, G. and Short, A.D., 1993.The effect of tide range on beach morphodynamics and morphology: a conceptual model. Journal of Coastal Research, 9, 785-800.

Morris, B.D., Davidson, M.A., Huntley, D.A., 2001.Measurements of the Response of a Coastal Inlet Using Video Monitoring Techniques. Marine Geology, 175, p.251-272.

Pilkey Jr, O.H., Neal, W.J., Monteiro, J.H., Dias, J.M.A., 1989. Algarve Barrier Islands: A noncoastal-Plain system in Portugal. Journal of Coastal Research, 5, 239-261.

Pires, O.H., 1998. Project INDIA. Preliminary report on wave climate at Faro. Instituto de meteorologica, IST, Lisbon, April 1998, 38p.

Schoonees, J.S. and Theron, A.K., 1993. Review of field database for longshore sediment transport. Coastal Engineering, 19, 1-25.

Vila, A., Dias, J.M.A., Ferreira, O. and Matias, A., 1999. Natural evolution of an artificial inlet. Proceedings of Coastal Sediments ' 99 - Long Island, New York, June 1999, pp. 1478-1488.

White, T. E., 1998. Status of measurement techniques for coastal sediment transport. Coastal Engineering, 35, pp. 17-45.

White, T.E. and Inman, D.L., 1989. Transport determination by tracers. In: Seymour, R.J. (ed.) Nearshore Sediment Transport, Plenum Press, pp.115-128.

Williams, J., Arens, B., Davidson, M., Dias, A., Howa, H., O'Connor, B., Sarmento, A. Voulgaris, G., 1998. "INDIA: Inlet Dynamics Initiative: Algarve", Proc.Oceans'98, 28/9-1/10, Nice France.

Zenkovitch, V. P. 1958. Fluorescent Substances as Tracers for Studying the Movement of Sand on the Sea Bed, Experiments Conducted in the U.S.S.R. Dock and Harbor Authority, 40: 280-283. 